\title{
Gray Matter Alterations in Obsessive-Compulsive Disorder: An Anatomic Likelihood Estimation Meta-Analysis
}

\author{
Jean-Yves Rotge*,1,2, Nicolas Langbour', Dominique Guehl 1,3, Bernard Bioulac ${ }^{1,3}$, Nematollah Jaafari ${ }^{4}$, \\ Michele Allard ${ }^{5,6,7}$, Bruno Aouizerate ${ }^{2}$ and Pierre Burbaud ${ }^{1,3}$ \\ 'Laboratoire Mouvement Adaptation Cognition, CNRS UMR 5227, Université Bordeaux 2, Bordeaux, France; ${ }^{2}$ Service Universitaire de \\ Psychiatrie, Centre Hospitalier Charles Perrens, Bordeaux, France; ${ }^{3}$ Senvice de Neurophysiologie Clinique, Centre Hospitalier Universitaire \\ Pellegrin, Bordeaux, France; ${ }^{4}$ CIC-P U 802, Senvice Hospitalo-Universitaire de Psychiatrie Adulte, Centre Hospitalier Henri Laborit, Centre \\ Hospitalier La Millétrie, Université de Poitiers, Poitiers, France; ${ }^{5}$ Laboratoire d'Imagerie Moléculaire et Fonctionnelle, CNRS UMR 523I, Université \\ Bordeaux 2, Bordeaux, France; ${ }^{6}$ Service de Médecine Nucléaire, Centre Hospitalier Universitaire Pellegrin, Bordeaux, France; ${ }^{7}$ Ecole Pratique des \\ Hautes Etudes, Bordeaux, France
}

Many voxel-based morphometry (VBM) studies have found abnormalities in gray matter density (GMD) in obsessive-compulsive disorder (OCD). Here, we performed a quantitative meta-analysis of VBM studies contrasting OCD patients with healthy controls (HC). A literature search identified 10 articles that included 343 OCD patients and $318 \mathrm{HC}$. Anatomic likelihood estimation meta-analyses were performed to assess GMD changes in OCD patients relative to HC. GMD was smaller in parieto-frontal cortical regions, including the supramarginal gyrus, the dorsolateral prefrontal cortex, and the orbitofrontal cortex, and greater in the basal ganglia (putamen) and the anterior prefrontal cortex in OCD patients relative to HC. No significant differences were found between children and adults. Our findings indicate differences in GMD in parieto-frontal areas and the basal ganglia between OCD patients and HC. We conclude that structural abnormalities within the prefrontal-basal ganglia network are involved in OCD pathophysiology.

Neuropsychopharmacology (2010) 35, 686-69I; doi:I 0.1038/npp.2009.175; published online 4 November 2009

Keywords: basal ganglia; meta-analysis; obsessive-compulsive disorder; parietal cortex; prefrontal cortex; voxel-based morphometry

\section{INTRODUCTION}

Obsessive-compulsive disorder (OCD) is a disabling anxiety disorder characterized by a chronic course. Over recent decades, several neuroimaging studies have contributed to identify changes in the brain that may mediate the disorder. For instance, functional abnormalities have been consistently described within frontosubcortical loops, which originate in the orbitofrontal cortex (OFC) (Menzies et al, 2008a).

A recently developed whole-brain technique, called voxelbased morphometry (VBM), allows voxel-wise between group comparisons of the local concentration of gray matter, often referred to as 'gray matter density' (GMD) (Ashburner and Friston, 2000). VBM allows researchers to locate structural abnormalities with high anatomic resolution, which contributes to the better understanding of OCD pathophysiology. However, results from VBM studies have remained inconsistent and controversial. For example, some studies have found that OCD patients have greater

*Correspondence: Dr J-Y Rotge, Laboratoire Mouvement Adaptation Cognition, CNRS UMR 5227, Université Bordeaux 2, 146 rue LéoSaignat, Bordeaux, Aquitaine 33076, France, Tel: + 33055757 I 5 5।, Fax: + 33055690 | 4 21, E-mail: jeanyves.rotge@mac.com Received 19 August 2009; revised 28 September 2009; accepted 29 September 2009
OFC compared with control subjects (Valente et al, 2005; Szeszko et al, 2008; Yoo et al, 2008), and some other studies reported a smaller OFC in OCD patients (Pujol et al, 2004; Christian et al, 2008; Lázaro et al, 2009). Thus, a quantitative overview of VBM findings in OCD is necessary.

Here, we conducted an anatomic likelihood estimation (ALE) meta-analysis of VBM studies contrasting OCD patients and healthy controls (HC). The ALE method is a powerful voxel-based meta-analytic technique, which was originally designed for not only functional neuroimaging studies (Turkeltaub et al, 2002), but it is also appropriate for VBM studies (Ellison-Wright et al, 2008; Glahn et al, 2008). The aim of the present meta-analysis was to identify brain areas implicated across published VBM studies in OCD. Furthermore, to assess neurodevelopmental GMD changes in OCD, we compared structural abnormalities between children and adults.

\section{MATERIALS AND METHODS}

\section{Literature Search and Study Selection}

We searched the MEDLINE and PsycINFO databases through April 2009, without limits on the year of publication, using the keywords 'obsessive compulsive disorder,' 'imaging,' 'neuroimaging,' 'structural imaging,' 'magnetic 
resonance imaging,' 'MRI,' 'morphometry,' or 'voxel-based morphometry.' The reference lists of identified articles and review articles were also searched to obtain additional papers. Studies were considered for inclusion if (1) they were published in English in a peer-reviewed journal, (2) they included subjects with a primary diagnosis of OCD on the basis of DSM-III, DSM-III-R, DSM-IV, or DSM-IV-R criteria, (3) they used the VBM method (Ashburner and Friston, 2000), (4) they compared an OCD group with an HC group, and (5) they reported whole-brain results and stereotactic $(x, y, z)$ coordinates of group differences. To ensure that data were not entered twice from a study population that had been analyzed in more than one publication, all articles written by a given research group were carefully scrutinized. In such cases, we systematically included data from the authors' largest study population and excluded others. In case in which we could not determine data originality, we contacted the corresponding author to determine whether there was subject overlap between multiple studies.

We identified 10 VBM studies in our database search. These studies included 343 OCD patients ( 80 children and 263 adults) and $318 \mathrm{HC}$ (69 children and 249 adults) (Pujol et al, 2004; Valente et al, 2005, Carmona et al, 2007; Christian et al, 2008; Szeszko et al, 2008; Gilbert et al, 2008a, b; Yoo et al, 2008; Lázaro et al, 2009; Van den Heuvel et al, 2009). Study selection was performed by one author (J-YR) and independently verified by another (NL).

\section{Data Extraction}

The identified articles included 66 foci (26 in children and 40 in adults), among which 23 corresponded to a greater gray matter (10 in children and 13 in adults) and 43 corresponded to a smaller gray matter (16 in children and 27 in adults) in OCD patients compared with HC. For each study, we identified the standardized atlas (Montreal Neurological Institute or Talairach spaces) and Standardized Precipitation Index ( $z$-scores or $t$-values) that was used. The Montreal Neurological Institute coordinates were transformed into Talairach coordinates using the icbm2tal algorithm (Lancaster et al, 2007). For each focus, we extracted the corresponding coordinates, volumes, and SPI values. Data extraction was performed by one author (J-YR) and independently verified by another (NL).

\section{ALE Meta-Analyses}

ALE analyses were completed using Scribe, Sleuth, and GingerALE software (BrainMap, University of Texas) (Lancaster et al, 2007; Laird et al, 2005a, b). Separate ALE maps were created for coordinates associated with greater or smaller GMD in OCD patients compared with HC. Additional ALE maps were created for samples from children and adults, and they were then compared. Analyses were performed after recommendations from BrainMap (http://www.brainmap.org). A full-width half-maximum of $12 \mathrm{~mm}$ was used. A permutation test of randomly distributed foci allowed the determination of the statistical significance of the resulting ALE values, corrected for multiple comparisons. Five thousand permutations were performed using the same full-width half-maximum value and the same number of foci used to compute ALE values. A conservative threshold for statistical significance was set at $p<0.05$ (false discovery rate corrected) with a minimum cluster size of $400 \mathrm{~mm}^{3}$. We used MRIcron software (http:// www.sph.sc.edu/comd/rorden/mricron/) to visualize ALE maps overlaid onto a high-resolution brain template generated by the International Consortium for Brain Mapping (Kochunov et al, 2002).

\section{RESULTS}

The ten identified VBM studies and the main characteristics of the included populations are presented in Table 1 . The results from ALE meta-analyses of VBM studies that included both children and adults are reported in Table 2 and depicted in Figure 1. GMD was smaller in many cortical areas, including the frontal eye fields (FEF) (Brodmann Area[BA] 8, superior frontal gyrus), supramarginal gyrus (BA 40), dorsolateral prefrontal cortex (DLPFC) (BA 9, middle frontal gyrus), and the medial part of the anterior prefrontal cortex (aPFC) (BA 10, medial frontal gyrus). GMD was greater in the putamen and in the lateral part of the OFC (BA 47, inferior frontal gyrus) in patients with OCD.

The results from ALE meta-analyses of children and adult samples are presented in Supplementary Materials (Supplementary Tables S1-2; Supplementary Figures S1-2). To assess differences in GMD with age, we compared the ALE maps generated for children and adult samples. No significant difference was found.

We next examined the effect of the statistical threshold chosen for the generation of ALE maps. The use of a more conservative threshold $(p<0.01)$ did not affect the results for any reported analysis, except that cluster volumes were generally smaller, requiring consideration of clusters under $400 \mathrm{~mm}^{3}$.

Finally, we conducted leave-one-out sensitivity analyses by repeating the analyses with the consecutive exclusion of each study to ensure that the overall results were not influenced by a single study. We found no marked differences regarding cerebral regions that showed GMD differences when using cluster size $<400 \mathrm{~mm}^{3}$. No significant differences were found when comparing each generated ALE map to the overall ALE map (Figure 1).

\section{DISCUSSION}

The present voxel-based meta-analysis pooled VBM studies that found structural changes in gray matter in 343 OCD patients. Our results showed that OCD patients exhibited smaller GMD in several parietal and frontal cortical areas and greater GMD in the basal ganglia and in the lateral part of the OFC. The findings from the present meta-analysis are consistent with currently accepted neurobiological models of OCD, which focus on dysfunction in OFC-striatal circuits. In a recent review, Menzies et al (2008a) showed that findings from anatomical and functional neuroimaging studies are compatible with those from cognitive studies. Furthermore, the authors outlined the involvement of areas other than the OFC and striatum in OCD, in particular the DLPFC and the parietal cortex. 
Table I Included Voxel-Based Morphometry Studies

\begin{tabular}{|c|c|c|c|c|c|c|c|}
\hline & Subjects & $\begin{array}{l}\text { Number of } \\
\text { subjects }\end{array}$ & $\begin{array}{c}\text { Age } \\
\text { mean (SD) }\end{array}$ & $\begin{array}{c}\% \text { of medicated } \\
\text { patients }\end{array}$ & $\begin{array}{l}\text { Y-BOCS score } \\
\text { mean (SD) }\end{array}$ & $\begin{array}{l}\text { Smaller } \\
\text { GMD }\end{array}$ & $\begin{array}{c}\text { Greater } \\
\text { GMD }\end{array}$ \\
\hline \multicolumn{8}{|l|}{ Samples from children } \\
\hline & $\mathrm{HC}$ & 18 & $13(3)$ & - & - & & \\
\hline Szeszko et al (2008) & OCD & 37 & $13(2.7)$ & 0 & $24.9(6.0)$ & $x$ & $x$ \\
\hline & $\mathrm{HC}$ & 10 & I3.4 (2.6) & - & - & & \\
\hline \multirow[t]{2}{*}{ Lázaro et al (2009) } & OCD & 15 & I3.7 (2.5) & 0 & $25.9(5.6)$ & & $x$ \\
\hline & $\mathrm{HC}$ & 15 & $\mid 4.3(2.5)$ & - & - & & \\
\hline \multicolumn{8}{|l|}{ Samples from adults } \\
\hline & $\mathrm{HC}$ & 15 & $32.3(11.8)$ & - & - & & \\
\hline \multirow[t]{2}{*}{ Gilbert et al (2008b) } & OCD & 25 & $37.5 \quad 10.7)$ & 80 & $26.9(6.3)$ & $x$ & $x$ \\
\hline & $\mathrm{HC}$ & 20 & $29.8(7.9)$ & - & - & & \\
\hline \multirow[t]{2}{*}{ Yoo et al (2008) } & OCD & 71 & $26.6(7.5)$ & 83.1 & $22.8(8.4)$ & $x$ & $x$ \\
\hline & $\mathrm{HC}$ & 71 & $26.7(6.1)$ & - & - & & \\
\hline \multirow[t]{2}{*}{ Christian et al (2008) } & OCD & 21 & $38(9.6)$ & 81 & $27.0(5.2)$ & $x$ & \\
\hline & $\mathrm{HC}$ & 21 & $38.9(9.8)$ & - & - & & \\
\hline \multirow[t]{2}{*}{ Van den Heuvel et al (2009) } & OCD & 55 & $33.7(9.2)$ & 0 & $22.8(6.1)$ & & $x$ \\
\hline & $\mathrm{HC}$ & 50 & $31.4(7.6)$ & - & - & & \\
\hline
\end{tabular}

OCD, obsessive-compulsive disorder; HC, healthy controls; Y-BOCS, yale-brown obsessive-compulsive scale; GMD, gray matter density; SD, standard deviation.

Table 2 Gray Matter Density Changes in all OCD Patients Relative to Healthy Controls

\begin{tabular}{|c|c|c|c|c|c|c|}
\hline \multirow{2}{*}{ Brain regions } & \multirow{2}{*}{$\begin{array}{l}\text { Brodmann } \\
\text { area }\end{array}$} & \multicolumn{3}{|c|}{ Talairach coordinates } & \multirow{2}{*}{$\begin{array}{l}\text { Volume } \\
\left(\mathrm{mm}^{3}\right)\end{array}$} & \multirow{2}{*}{$\begin{array}{c}\text { Maximum } \\
\text { ALE value }\left(\times 10^{3}\right)\end{array}$} \\
\hline & & $x$ & $y$ & $\mathbf{z}$ & & \\
\hline \multicolumn{7}{|l|}{ Gray matter density reduction } \\
\hline R superior frontal gyrus & 8 & 21 & 24 & 47 & 1152 & 6.4 \\
\hline $\mathrm{L}$ middle frontal gyrus & 9 & -35 & 26 & 36 & 960 & 5.2 \\
\hline $\mathrm{R}$ middle frontal gyrus & 9 & 41 & 33 & 30 & 536 & 4.0 \\
\hline R supramarginal gyrus & 40 & 57 & -44 & 33 & 424 & 4.1 \\
\hline \multicolumn{7}{|c|}{ Gray matter density enhancement } \\
\hline L inferior frontal gyrus & 47 & -21 & 18 & -14 & 1120 & 4.1 \\
\hline
\end{tabular}

$L$, left; $R$, right; $A L E$, anatomic likelihood estimation.

Recently, a prior meta-analysis of ROI volume studies in OCD reported a smaller volume in the OFC, in the anterior cingulate cortex (ACC), and a higher volume in the thalamus, but no change in the basal ganglia (Rotge et al, 2009). The results from this ROI meta-analysis seem to be in contrast to the present findings from a VBM meta-analysis.
There may be many reasons for such discrepancies. First, there were a larger number of studies included in the ROI meta-analysis than in the VBM meta-analysis, which may minimize the risk of false negatives in ROI studies. Second, clinical samples from studies included in the ROI meta-analysis differ from those included in the VBM 


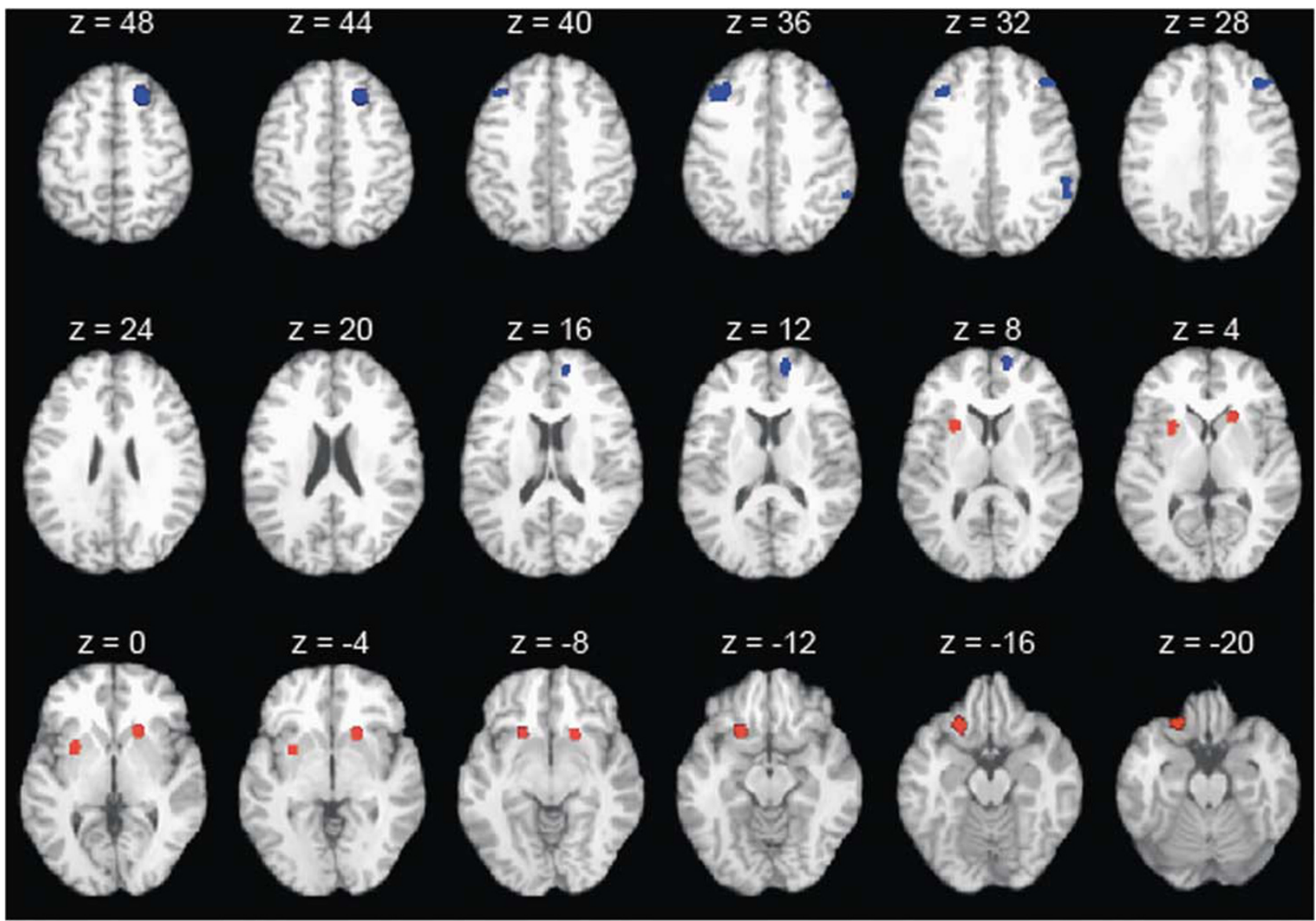

Figure I ALE map investigating differences in GMD between OCD patients and healthy subjects. Regions in blue are associated with a smaller gray matter in OCD patients relative to HC. Regions in red are associated with greater gray matter in OCD patients relative to HC. Smaller gray matter was found in the right superior frontal gyrus $(z=48,44)$, left middle frontal gyrus $(z=40,36,32)$, right medial frontal gyrus $(z=16,12,8)$, right middle frontal gyrus $(z=32,28)$, and the right supramarginal gyrus $(z=36,32)$. Greater gray matter was found in the left inferior frontal gyrus $(z=-12,-16,-20)$, right putamen $(z=4,0,-4,-8)$, and the left putamen $(z=8,4,0,-4,-8)$.

meta-analysis. For example, the ROI meta-analysis mainly includes adult studies, whereas $40 \%$ of the studies of VBM meta-analysis corresponded to samples from children. Finally and most importantly, methodological differences between ROI and VBM techniques may account for these discrepancies. Indeed, VBM is useful for identifying brain abnormalities within whole brains, but factors other than volume alone may influence VBM results. For example, changes in the shape or displacement of structures in the course of spatial normalization may affect the final results (Kubicki et al, 2002). However, VBM studies and the present meta-analysis extend results from the prior ROI meta-analysis and provide new information regarding structural alterations in OCD. This is especially true for brain regions that have been poorly investigated in ROI studies, such as the parietal cortex or DLPFC, and for subterritories of a given brain region, such as the prefrontal cortex.

Smaller GMD was identified in the FEF, DLPFC, supramarginal gyrus, and aPFC. These structural changes may be related to neuropsychological impairments described in OCD patients. Spengler et al (2006) described oculomotor impairments and a higher frequency of anticipatory saccades in OCD patients relative to $\mathrm{HC}$, suggesting the existence of dysfunctional events in the FEF of OCD patients. Furthermore, there is evidence for dysfunction of the DLPFC in OCD. Impairments in executive functions, such as planning, have been described in OCD patients. These deficits were associated with decreased activation in this cortical area when compared with HC (Van den Heuvel et al, 2005). Smaller GMD found in the supramarginal gyrus, a part of the parietal cortex, is consistent with diffusion tensor imaging studies (Menzies et al, 2008b) that showed white matter abnormalities in this brain region and with functional imaging studies that showed a negative relationship between regional cerebral blood flow and symptom intensity (McGuire et al, 1994). These findings suggest that the supramarginal gyrus may have a function in the ability of patients to resist the obsessive processes. However, the functional contribution of this cortical area to OCD remains unclear and further studies are required to investigate its function in OCD pathophysiology. Finally, GMD was also found to be smaller within the aPFC. Many neuroimaging studies have shown that this cortical area is involved in multiple cognitive tasks. However, when examining commonalities across studies, Ramnani and Owen (2004) have proposed that the aPFC should contribute to the coordination of information 
processing and information transfer between multiple operations across the supramodal cortex. In regard to OCD phenomenology, it could be assumed that dysfunction of the aPFC could play a role in deficits in cognitive flexibility, in particular in cognitive reallocation, which may contribute to maintenance of repetitive behaviors. However, the specific function of the aPFC in OCD remains unknown and awaits clarification.

Greater GMD was identified in the bilateral putamen, which is a part of the striatum. This finding is consistent with neurobiological models of OCD and with functional neuroimaging studies suggesting that dysfunction in the putamen contributes to the pathogenesis of OCD (Menzies et al, 2008a; Rauch et al, 1997). Furthermore, OCD symptom severity was associated with metabolic activity in the putamen (Kwon et al, 2003), whereas decreased metabolic activity in the putamen after pharmacological treatment was associated with an improvement in cognitive function in OCD patients (Kang et al, 2003). GMD was also found to be greater in the lateral part of the OFC in OCD patients compared with HC. The OFC is thought to have a function in several cognitive processes, such as the selection, comparison, and judgment of motivationally salient stimuli, and in the motivational aspects of decision making. In particular, the OFC has a function in error prediction by encoding the representation of the value of an expected outcome, which is used to anticipate the positive and negative consequences that are likely to follow a given action (O'Doherty, 2007; Wallis, 2007). The OFC also increases the evaluation of the occurrence of negative consequences that may follow an action in OCD and, therefore, mediates obsessive thoughts that lead to repetitive or ritualistic behaviors intended to prevent negative consequences as a way to relieve anxiety. The OFC is likely related to deficits of cognitive regulation of emotionally salient information. Accordingly, Chamberlain et al (2008)recently showed that, in comparison with HC, lateral OFC activation is reduced during a task that assesses behavioral flexibility in OCD patients and in unaffected relatives. These findings suggest that the lateral OFC could have a central function in cognitive flexibility deficits and, thus, in the genesis of pathological habits (Chamberlain et al, 2008).

In the present meta-analysis, we failed to show any significant differences in GMD between children and adults. This was an unexpected result because many factors should reasonably contribute to GMD changes with aging. First, children and adults differ for different clinical variables, such as illness duration, age of onset, or treatment intervention, which may potentially influence neuroimaging findings. Second, there are several lines of evidence in the literature suggesting that OCD is a neurodevelopmental disorder. Rosenberg and Keshavan (1998) proposed that OCD may be underlined by 'a developmentally mediated network dysplasia in prefrontal cortical circuits'. This neurodevelopmental view may be supported by differences in GMD between children and adults. One possible explanation for the absence of any significant differences between pediatric and adult samples may be the low number of foci, which may contribute to false negative results. Further hypothesis-driven ROI studies could be helpful to resolve this issue.
The present meta-analysis has several limitations. First, our meta-analysis is subject to publication bias. Studies that failed to show significant effects may not have been published. In the present meta-analysis, we identified only 10 VBM studies and we did not identify any articles without an effect. Second, we failed to consider clinical variables, such as symptom dimensions, medication status, comorbidity status, and symptom severity scores. Although these variables may be associated with differential structural abnormalities, the low number of VBM studies did not allow us to control for these factors. However, the absence of marked difference when consecutively excluding each study argues in favor of the robustness of our findings despite the clinical heterogeneity of study populations.

In conclusion, the present meta-analysis of VBM studies showed that OCD patients exhibited gray matter abnormalities in parieto-frontal cortical areas and in the basal ganglia. Further structural neuroimaging studies are required to assess gray matter changes associated with clinical correlates. Furthermore, functional studies may be helpful to elucidate the specific function of these areas in the genesis of OCD symptoms.

\section{ACKNOWLEDGEMENTS}

J-Y Rotge was supported by a grant from the Fondation pour la Recherche Médicale (Medical Research Foundation).

\section{DISCLOSURE}

The authors declare no conflict of interest.

\section{REFERENCES}

Ashburner J, Friston KJ (2000). Voxel-based morphometry-the methods. NeuroImage 11: 805-821.

Carmona S, Bassas N, Rovira M, Gispert JD, Soliva JC, Prado M et al (2007). Pediatric OCD structural brain deficits in conflic monitoring circuits: a voxel-based morphometry study. Neurosci Lett 421: 218-223.

Chamberlain SR, Menzies L, Hampshire A, Suckling J, Fineberg $\mathrm{NA}$, del Campo $\mathrm{N}$ et al (2008). Orbitofrontal dysfunction in patients with obsessive-compulsive disorder and their unaffected relatives. Science 321: 421-422.

Christian CJ, Lencz T, Robinson DG, Burdick KE, Ashtari M, Malhotra AK et al (2008). Gray matter structural alterations in obsessive-compulsive disorder: relationship to neuropsychological functions. Psychiatry Res 164: 123-131.

Ellison-Wright I, Glahn DC, Laird AR, Thelen SM, Bullmore ET (2008). The anatomy of first-episode and chronic schizophrenia: an anatomical likelihood estimation meta-analysis. $\mathrm{Am} \mathrm{J}$ Psychiatry 165: 1015-1023.

Gilbert AR, Keshavan MS, Diwadkar V, Nutche J, Macmaster F, Easter PC et al (2008a). Gray matter differences between pediatric obsessive-compulsive disorder patients and high-risk siblings: a preliminary voxel-based morphometry study. Neurosci Lett 435: 45-50.

Gilbert AR, Mataix-Cols D, Almeida JR, Lawrence N, Nutche J, Diwadkar V et al (2008b). Brain structure and symptom dimension relationships in obsessive-compulsive disorder: a voxel-based morphometry study. J Affect Disord 109: 117-126.

Glahn DC, Laird AR, Ellison-Wright I, Thelen SM, Robinson JL, Lancaster JL et al (2008). Meta-analysis of gray matter anomalies 
in schizophrenia: application of anatomic likelihood estimation and network analysis. Biol Psychiatry 64: 774-781.

Kang DH, Kwon JS, Kim JJ, Youn T, Park HJ, Kim MS et al (2003). Brain glucose metabolic changes associated with neuropsychological improvements after 4 months of treatment in patients with obsessive-compulsive disorder. Acta Psychiatr Scand 107: 291-297.

Kochunov P, Lancaster J, Thompson P, Toga AW, Brewer P, Hardies $J$ et al (2002). An optimized individual target brain in the Talairach coordinate system. NeuroImage 17: 922-927.

Kubicki M, Shenton ME, Salisbury DF, Hirayasu Y, Kasai K, Kikinis $\mathrm{R}$ et al (2002). Voxel-based morphometric analysis of gray matter in first episode schizophrenia. NeuroImage 17: 1711-1719.

Kwon JS, Kim JJ, Lee DW, Lee JS, Lee DS, Kim MS et al (2003). Neural correlates of clinical symptoms and cognitive cognitive dysfunctions in obsessive-compulsive disorder. Psychiatry Res 122: 37-47.

Laird AR, Fox M, Price CJ, Glahn DC, Uecker AM, Lancaster JL et al (2005a). ALE meta-analysis: controlling the false discovery rate and performing statistical contrasts. Hum Brain Mapp 25: 155-164.

Laird AR, Lancaster JL, Fox PT (2005b). BrainMap: the social evolution of a functional neuroimaging database. Neuroinformatics 3: 65-78.

Lancaster JL, Tordesillas-Gutierrez D, Martinez M, Salinas F, Evans A, Zilles K et al (2007). Bias between MNI and Talairach coordinates analyzed using the ICBM-152 brain template. Hum Brain Mapp 28: 1194-1205.

Lázaro L, Bargalló N, Castro-Fornieles J, Falcón C, Andrés S, Calvo $\mathrm{R}$ et al (2009). Brain changes in children and adolescents with obsessive-compulsive disorder before and after treatment: a voxel-based morphometric MRI study. Psychiatry Res 172: 140-146.

McGuire PK, Bench CJ, Frith CD, Marks IM, Frackowiak RS, Dolan RJ (1994). Functional anatomy of obsessive-compulsive phenomena. Br J Psychiatry 164: 459-468.

Menzies L, Chamberlain SR, Laird AR, Thelen SM, Sahakian BJ, Bullmore ET (2008a). Integrating evidence from neuroimaging and neuropsychological studies of obsessive-compulsive disorder: the orbitofronto-striatal model revisited. Neurosci Biobehav Rev 32: 525-549.

Menzies L, Williams GB, Chamberlain SR, Ooi C, Fineberg N, Suckling J et al (2008b). White matter abnormalities in patients with obsessive-compulsive disorder and their first-degree relatives. Am J Psychiatry 165: 1308-1315.

O'Doherty JP (2007). Lights, camembert, action! The role of human orbitofrontal cortex in encoding stimuli, rewards, and choices. Ann NY Acad Sci 1121: 254-272.
Pujol J, Soriano-Mas C, Alonso P, Cardoner N, Menchón JM, Deus $J$ et al (2004). Mapping structural brain alterations in obsessivecompulsive disorder. Arch Gen Psychiatry 61: 720-730.

Ramnani N, Owen AM (2004). Anterior prefrontal cortex: insights into function from anatomy and neuroimaging. Nat Rev Neurosci 5: 184-194.

Rauch SL, Savage CR, Alpert NM, Dougherty D, Kendrick A, Curran T et al (1997). Probing striatal function in obsessivecompulsive disorder: a PET study of implicit sequence learning. J Neuropsychiatry Clin Neurosci 9: 568-573.

Rosenberg DR, Keshavan MS (1998). Towar a neurodevelopmental model of obsessive-compulsive disorder. Biol Psychiatry 43: 623-640.

Rotge JY, Guehl D, Dilharreguy B, Tignol J, Bioulac B, Allard M et al (2009). Meta-analysis of brain volume changes in obsessivecompulsive disorder. Biol Psychiatry 65: 75-83.

Spengler D, Trillenberg P, Sprenger A, Nagel M, Kordon A, Junghanns $\mathrm{K}$ et al (2006). Evidence from increased anticipation of predictive saccades for a dysfunction of fronto-striatal circuits in obsessive-compulsive disorder. Psychiatry Res 143: 77-88.

Szeszko PR, Christian C, MacMaster F, Lencz T, Mirza Y, Taormina SP et al (2008). Gray matter structural alterations in psychotropic drug-naïve pediatric obsessive-compulsive disorder: an optimized voxel-based morphometry study. Am J Psychiatry 165: 1299-1307.

Turkeltaub PE, Eden GF, Jones KM, Zeffiro TA (2002). Metaanalysis of the functional neuroanatomy of single-word reading: method and validation. NeuroImage 16: 765-780.

Valente AA, Miguel EC, Castro CC, Amaro E, Duran FLS, Buchpiguel CA et al (2005). Regional gray matter abnormalities in obsessive-compulsive disorder: a voxel-based morphometry study. Biol Psychiatry 58: 479-487.

Van den Heuvel OA, Remijnse PL, Mataix-Cols D, Vrenken H, Groenewegen HJ, Uylings HB et al (2009). The major symptom dimensions of obsessive-compulsive disorder are mediated by partially distinct neural systems. Brain 132: 853-868.

Van den Heuvel OA, Veltman DJ, Groenewegen HJ, Cath DC, van Balkom AJ, van Hartskamp J et al (2005). Frontal-striatal dysfunction during planning in obsessive-compulsive disorder. Arch Gen Psychiatry 62: 301-309.

Wallis JD (2007). Orbitofrontal cortex and its contribution to decision-making. Annu Rev Neurosci 30: 31-56.

Yoo SY, Roh MS, Choi JS, Kang DH, Ha TH, Lee JM et al (2008). Voxel-based morphometry study of gray matter abnormalities in obsessive-compulsive disorder. J Korean Med Sci 23: $24-30$.

Supplementary Information accompanies the paper on the Neuropsychopharmacology website (http://www.nature.com/npp) 